\title{
Extending the vulnerability-stress model of mental disorders: three-dimensional NPSR1 $\times$ environment $\times$ coping interaction study in anxiety
}

Miriam A. Schiele, Katharina Herzog, Leonie Kollert, Christoph Schartner, Elisabeth J. Leehr, Joscha Böhnlein, Jonathan Repple, Karoline Rosenkranz, Tina B. Lonsdorf, Udo Dannlowski, Peter Zwanzger, Andreas Reif, Paul Pauli, Jürgen Deckert and Katharina Domschke

\section{Background}

The general understanding of the 'vulnerability-stress model' of mental disorders neglects the modifying impact of resilienceincreasing factors such as coping ability.

\section{Aims}

Probing a conceptual framework integrating both adverse events and coping factors in an extended 'vulnerability-stress-coping model' of mental disorders, the effects of functional neuropeptide S receptor gene (NPSR1) variation (G), early adversity (E) and coping factors $(C)$ on anxiety were addressed in a three-dimensional $\mathrm{G} \times \mathrm{E} \times \mathrm{C}$ model

\section{Method}

In two independent samples of healthy probands (discovery: $n=1403$; replication: $n=630$ ), the interaction of NPSR1 rs324981, childhood trauma (Childhood Trauma Questionnaire, CTQ) and general self-efficacy as a measure of coping ability (General Self-Efficacy Scale, GSE) on trait anxiety (State-Trait Anxiety Inventory) was investigated via hierarchical multiple regression analyses.

\section{Results}

In both samples, trait anxiety differed as a function of NPSR1 genotype, CTQ and GSE score (discovery: $\beta=0.129, P=3.938 \times 10^{-8}$; replication: $\beta=0.102, P=0.020$ ). In A allele carriers, the relationship between childhood trauma and anxiety was moderated by general self-efficacy: higher self-efficacy and childhood trauma resulted in low anxiety scores, and lower self-efficacy and childhood trauma in higher anxiety levels. In turn, TT homozygotes displayed increased anxiety as a function of childhood adversity unaffected by general self-efficacy.

\section{Conclusions}

Functional NPSR1 variation and childhood trauma are suggested as prime moderators in the vulnerability-stress model of anxiety, further modified by the protective effect of self-efficacy. This $G \times$ $\mathrm{E} \times \mathrm{C}$ approach - introducing coping as an additional dimension further shaping a $\mathrm{G} \times \mathrm{E}$ risk constellation, thus suggesting a three-dimensional 'vulnerability-stress-coping model' of mental disorders - might inform targeted preventive or therapeutic interventions strengthening coping ability to promote resilient functioning

\section{Keywords}

Resilience; general self-efficacy; differential susceptibility; neuropeptide $\mathrm{S}$ receptor; anxiety disorder.

\section{Copyright and usage}

(c) The Author(s), 2020. Published by Cambridge University Press on behalf of the Royal College of Psychiatrists. This is an Open Access article, distributed under the terms of the Creative Commons Attribution-NonCommercial-NoDerivatives licence (http://creativecommons.org/licenses/by-nc-nd/4.0/), which permits non-commercial re-use, distribution, and reproduction in any medium, provided the original work is unaltered and is properly cited. The written permission of Cambridge University Press must be obtained for commercial re-use or in order to create a derivative work.
Mental disorders are characterised by a complex-genetic aetiology, where - assuming the traditional 'vulnerability-stress model' - biological factors interact with negative environmental influences to shape the risk towards the respective disorder. ${ }^{1}$ Translating this concept to current methodology, research focusing on gene-environment $(\mathrm{G} \times \mathrm{E})$ interactions has paid much attention to the interactive detrimental effects of genetic risk factors and environmental adversity (for review see, for example, Musci et $\mathrm{al}^{2}$ ). The ability to successfully cope in the face of adversity, i.e. to 'successfully evoke the capacities of the organism to contend with problems', ${ }^{1}$ constitutes another key factor in shaping the risk-resilience spectrum towards mental disorders. ${ }^{3}$ However, apart from a few and mostly theoretical publications in schizophrenia research, ${ }^{4}$ coping has not been included in the general conception of the vulnerability-stress model and has not yet been systematically studied in synopsis with genetic and adverse environmental factors in mental disorders. Consequently, there is a need for probing a novel conceptual framework integrating both adverse events and coping factors in an extended 'vulnerability-stress-coping model' of mental disorders. In the present study, a three-dimensional $\mathrm{G} \times \mathrm{E} \times \mathrm{C}$ model expanded by the dimension of coping ability ('C') was therefore exemplarily applied to the phenotype of anxiety based on the following state of knowledge.

\section{The neuropeptide S system}

On a genetic level ('G'), the neuropeptide S (NPS) system constitutes one of the most promising biological candidates related to arousal and anxiety. The gene coding for the NPS receptor (NPSR1) is located on chromosome 7p14 and contains an A/T (Asn107Ile) single nucleotide polymorphism (rs324981), with the T allele resulting in a tenfold increase in NPS potency at the NPS receptor. ${ }^{5}$ This more active $\mathrm{T}$ allele has been linked to panic disorder per $s^{6}$ as well as to panic disorder endophenotypes such as elevated levels of anxiety sensitivity, ${ }^{6,7}$ enhanced response inhibition and increased error monitoring, ${ }^{7}$ increased heart rate and higher symptom reports during a behavioural avoidance test, ${ }^{6}$ and enhanced neuroendocrine and subjective responses to acute stress. ${ }^{8}$ On a neural level, $\mathrm{T}$ allele carriers exhibited increased amygdala activation in response to fearful and angry faces in healthy probands, ${ }^{9}$ whereas in patients with panic disorder the $\mathrm{T}$ allele appears to be related to attenuated activation of the dorsolateral prefrontal, lateral 
orbitofrontal and anterior cingulate cortex during the processing of fearful faces, ${ }^{6}$ potentially based on a delayed maturation of corticolimbic connectivity during adolescence. ${ }^{10}$ Taken together, the NPSR1 rs324981 variant has been shown to play a significant role in anxiety and anxiety disorders, particularly panic disorder and is involved in the modulation of a variety of intermediate anxiety phenotypes.

\section{Environment and coping}

On an environmental level ('E'), experiences of threat, interpersonal conflicts or stressful life events in adulthood as well as traumatic experiences in the sensitive period of childhood have been reported to constitute risk factors for the later development of anxiety disorders. ${ }^{11}$

Regarding the dimension of coping ability ('C'), self-efficacy referring to an individual's belief in his or her own ability to successfully cope with challenge and measured as a general construct termed general self-efficacy ${ }^{12}$ - constitutes a promising dimension related to coping with adversity. High general self-efficacy has been linked to lower levels of trait anxiety in healthy adolescents and adults, ${ }^{13,14}$ less severe symptoms of depression, ${ }^{14,15}$ decreased risk for symptoms of social anxiety in childhood ${ }^{16}$ and resilience against the deleterious effects of daily stressors, ${ }^{17}$ thus highlighting its function as an important link between environmental adversity and anxiety-related mental health outcomes.

\section{Gene-environment interactions}

In first $\mathrm{G} \times \mathrm{E}$ studies, the interactive effects of NPSR1 rs324981 genotype and adverse environmental aspects on anxiety-related phenotypes were described for childhood maltreatment, ${ }^{18}$ family adversity ${ }^{19}$ and urban upbringing. ${ }^{20}$ However, results differ regarding the allelic direction of this interaction: the TT genotype has been shown to drive anxiety risk dependent on experiences of childhood trauma ${ }^{18}$ and to confer enhanced amygdala activation during stress exposure in the context of high early urbanicity. ${ }^{20}$ By contrast, Laas et $\mathrm{al}^{19}$ observed an interaction of the AA genotype with a history of stressful life events conferring increased trait anxiety as well as with family adversity resulting in a higher risk for anxiety disorders. No study so far has looked into beneficial factors such as coping ability possibly further shaping the spectrum of risk and resilience towards anxiety and thus potentially reconciling previous diverging findings.

Against this background, the present study investigated the interactive effect of the functional NPSR1 rs324981 A/T variant, childhood trauma and general self-efficacy on trait anxiety in a large discovery and an independent replication sample of healthy volunteers applying a $\mathrm{G} \times \mathrm{E} \times \mathrm{C}$ approach in order to probe an extended 'vulnerability-stress-coping model' of mental disorders.

\section{Method}

\section{Samples and procedures}

The discovery sample included a total of 1403 healthy participants (women $n=826$, mean age 25.59 years, s.d. $=5.71$ ) and was recruited in the context of project Z02 within the Collaborative Research Centre SFB-TRR58 'Fear, Anxiety, Anxiety Disorders' during the project's second funding period at the Universities of Würzburg, Hamburg and Münster, Germany, between 2013 and 2016 (see Schiele et $\mathrm{al}^{21}$ ).

The replication sample, consisting of 630 healthy adults (women $n=445$, mean age 25.03 years, s.d. $=5.57$ ), was independently recruited during the above-mentioned project's third funding period at the Universities of Würzburg, Hamburg and Münster, Germany, between 2016 and 2018.
For both samples, inclusion criteria were defined as ethnic White descent (self-report up to third generation), age at inclusion between 18 and 50 years, right-handedness (see below) and fluency in German. Probands with a past or current DSM-IV axis I disorder as ascertained by experienced psychologists (Mini-International Psychiatric Interview), ${ }^{22}$ past or current severe neurological or somatic disorders, currently taking centrally active medication, excessive alcohol ( $\geq 15$ units/week), nicotine ( $\geq 20$ cigarettes/day) and caffeine ( $\geq 4$ cups/day) consumption, utilisation of illegal drugs, or pregnancy were excluded. Participants completed a set of questionnaires (see below). For genetic analyses, a venous blood sample $(\sim 18 \mathrm{~mL})$ was taken. The project has been designed to also provide a recruitment pipeline channelling comprehensively pheno- and genotyped participants into several subprojects within the SFB-TRR58 for specific research questions including neuroimaging studies (hence left-handedness was defined as an exclusion criterion).

Participation was remunerated with $50 €$. Written informed consent was obtained from all participants. The study was reviewed and approved by the ethical committees of the Universities of Würzburg, Hamburg and Münster and was conducted in compliance with the Declaration of Helsinki.

\section{Self-report measures}

The short form of the Childhood Trauma Questionnaire $\left(\mathrm{CTQ}^{23}\right)$, containing 28 items, retrospectively assesses the frequency of childhood maltreatment. The total score is obtained as the sum of all items, resulting in possible sum scores between 25 and 128 .

The General Self-Efficacy Scale $\left(\mathrm{GSE}^{24}\right)$ comprises ten items to assess perceived self-efficacy, i.e. a person's belief in their own ability to cope with difficulties, which can be summed up, resulting in total scores between 10 and 40 points.

The trait scale of the State-Trait Anxiety Inventory (STAI-T ${ }^{25}$ ) consists of 20 items capturing anxiety as a stable, trans-situational disposition. Seven items are reverse-coded to minimise errors because of arbitrary responding. After re-coding of the respective items, the total STAI-T score is calculated as the sum of all items, resulting in possible scores between 20 and 80 .

\section{Genotyping}

For genotyping of NPSR1 rs324981, DNA extracted from EDTA (ethylenediamine tetraacetic acid)-blood was amplified by polymerase chain reaction $\left(\mathrm{PCR}, 45 \mathrm{~s}\right.$ at $95^{\circ} \mathrm{C}, 45 \mathrm{~s}$ at $58^{\circ} \mathrm{C}, 45 \mathrm{~s}$ at $72^{\circ} \mathrm{C}$ for 35 cycles) using oligonucleotide primers F: $5^{\prime}$-TGCTTTGCATTTC CTCAGTG- $3^{\prime}$ and R: $5^{\prime}$-TTGTCTCATCACATTTGGAAGG-3'. PCR products were digested with AseI at $37^{\circ} \mathrm{C}$ overnight, separated on $3 \%$ agarose gel containing ethidium bromide, and visualised by ultraviolet light.

Hardy-Weinberg criteria as determined by the online program DeFinetti (http://ihg.gsf.de/cgi-bin/hw/hwal.pl) were fulfilled for both samples (discovery sample: TT, 306; AT, 710; AA, 387; $P=$ 0.592; replication sample: TT, 131; AT, 328; AA 171; $P=0.264)$. For further analyses, NPSR1 rs324981 genotypes were grouped into TT genotype versus A allele (AA/AT) carriers in accordance with previous studies in healthy probands. ${ }^{18}$

\section{Statistical analyses}

Group differences regarding continuous variables were analysed by means of $t$-tests. Differences regarding categorical variables were tested by means of $\chi^{2}$-tests. Possibly confounding gene-environment correlations were evaluated using Pearson's correlations. The influence of NPSR1 genotype, CTQ and GSE scores, as well as their interactions on STAI-T scores were tested via hierarchical 
multiple regression analyses (cf. Schiele et $\mathrm{al}^{21}$ ). Genotype groups were centred ${ }^{26}$ and coded as 0.5 (TT) and -0.5 (AA/AT). CTQ and GSE sum scores were centred (mean 0, s.d. $=1$ ). Regression analyses were performed in three steps: in the first step, main effects were entered into the model, i.e. genotype, centred CTQ sum score and centred GSE sum score. Gender (centred; 0.5, women; -0.5 , men) was entered as an additional factor in the first step. In a second step, all two-way interaction terms - genotype $x$ $\mathrm{CTQ}$, genotype $\times$ GSE and CTQ $\times$ GSE - were included. Finally, in a third step, the three-way interaction term comprising genotype $\times$ CTQ $\times$ GSE was added. All statistical tests were performed using SPSS V24 (SPSS Inc, Chicago, Illinois, USA). The significance level was set at 0.05 .

\section{Results}

\section{Descriptives}

Descriptive characteristics of the discovery and the replication sample are given in Table 1.

Genotype groups did not differ with regard to age (discovery sample: $t=1.13$, d.f. $=1401, P=0.258$; replication sample: $t=1.72$, d.f. $=628, P=0.125$ ), gender (discovery sample: $\chi^{2}=0.08$, d.f. $=1$, $P=0.777$; replication sample: $\chi^{2}=0.93$, d.f. $=1, P=0.335$ ), CTQ score (discovery sample: $t=-0.33$, d.f. $=1401, P=0.745$; replication sample: $t=-0.79$, d.f. $=628, P=0.431$ ), GSE score (discovery sample: $t=-1.45, \quad$ d.f. $=1401, \quad P=0.149$; replication sample: $t=-0.50$, d.f. $=628, P=0.619$ ), or STAI-T score (discovery sample: $t=-0.15$, d.f. $=1401, P=0.878$; replication sample: $t=0.85$, d.f. $=$ $628, P=0.395)$.

No significant gene-environment correlations with either predictor variable were observed (discovery sample: all $P \geq 0.149$; replication sample: all $P \geq 0.431$ ).

\section{Discovery sample}

Step 1 yielded significant main effects of CTQ score $(\beta=0.214$, $\left.t=9.960, \quad P=1.265 \times 10^{-22}\right)$ and GSE score $(\beta=-0.543$, $\left.t=-25.148, P=1.942 \times 10^{-115}\right)$. In step 2, significant main effects emerged for CTQ score $\left(\beta=0.214, t=8.213, P=4.976 \times 10^{-16}\right)$ and GSE score $\left(\beta=-0.566, t=-21.136, P=3.048 \times 10^{-86}\right)$. Step 3 returned significant main effects of CTQ score $(\beta=0.250$, $\left.t=9.405, \quad P=2.058 \times 10^{-20}\right)$ and GSE score $(\beta=-0.583$, $\left.t=-21.849, P=3.031 \times 10^{-91}\right)$, significant two-way interactions of GSE $\times$ CTQ scores $\left(\beta=0.086, t=3.669, P=2.250 \times 10^{-4}\right.$ and genotype $\times$ GSE score $(\beta=-0.056, t=-2.128, P=0.034)$ as well as a significant three-way interaction term of genotype $\times$ CTQ $\times$ GSE score $\left(\beta=0.129, t=5.524, P=3.938 \times 10^{-8}\right)$. The addition of the three-way interaction term in step 3 accounted for a significant increment in explained variance $\left(R^{2}=0.380, \Delta R^{2}=0.014\right.$, $\left.\Delta F=30.520, P=3.938 \times 10^{-8}\right)$.

\section{Table 1 Descriptive sample characteristics}

\begin{tabular}{|lcr|} 
& $\begin{array}{c}\text { Discovery sample } \\
(n=1403)\end{array}$ & $\begin{array}{c}\text { Replication sample } \\
(n=630)\end{array}$ \\
\hline Age, mean (s.d.) & $25.59(5.71)$ & $25.03(5.57)$ \\
Gender, women: $n$ (\%) & $826(58.9)$ & $445(70.6)$ \\
CTQ score, mean (s.d.) & $32.02(7.84)$ & $31.12(7.84)$ \\
GSE score, mean (s.d.) & $29.92(3.74)$ & $29.97(3.64)$ \\
STAI-T score, mean (s.d.) & $34.60(8.20)$ & $33.27(7.71)$ \\
NPSR1 rs324981 & & \\
TT & 306 & 131 \\
AA/AT & 1097 & 499 \\
CTQ, Childhood Trauma Questionnaire, GSE, General Self-Efficacy Scale; STAl-T: Trait \\
scale of the State-Trait Anxiety Inventory; NPSR1: neuropeptide S receptor gene. \\
\hline
\end{tabular}

The relationship between NPSR1 genotype, CTQ and GSE on STAI-T scores is depicted in Fig. 1(a); in A allele carriers, high general self-efficacy exerted a buffering effect on trait anxiety despite a history of childhood trauma, whereas low general self-efficacy led to increased anxiety scores in individuals with an otherwise equal genetic and environmental risk constellation. In turn, increases in trait anxiety as a function of childhood maltreatment were observed in TT homozygotes largely unaffected by general self-efficacy.

\section{Replication sample}

Significant main effects of CTQ $\left(\beta=0.251, t=7.661, P=7.041 \times 10^{-14}\right)$ and GSE score $\left(\beta=-0.511, t=-15.467, P=6.235 \times 10^{-46}\right)$ emerged in step 1. Step 2 returned significant main effects of CTQ $(\beta=0.224$, $\left.t=5.177, \quad P=3.043 \times 10^{-7}\right)$ and GSE score $(\beta=-0.520, \quad t=$ $\left.-11.552, P=4.230 \times 10^{-28}\right)$. In step 3 , significant main effects of CTQ $\left(\beta=0.260, t=5.677, P=2.109 \times 10^{-8}\right)$ and GSE score $(\beta=-0.518$, $\left.t=-11.546, \quad P=4.519 \times 10^{-28}\right)$ were obtained, as well as a significant three-way interaction term of genotype $\times \mathrm{CTQ} \times \mathrm{GSE}$ $(\beta=0.102, t=2.328, P=0.020)$. The addition of the three-way interaction term in step 3 accounted for a significant increment in explained variance $\left(R^{2}=0.341, \Delta R^{2}=0.006, \Delta F=5.421, P=0.020\right)$ (see Fig. 1(b)). Again, low or high GSE score, respectively, went along with increased or decreased trait anxiety depending on CTQ scores in A allele carriers, but not in TT homozygotes.

\section{Discussion}

\section{Main findings}

The present $\mathrm{G} \times \mathrm{E} \times \mathrm{C}$ study demonstrates a moderating influence of the coping factor 'general self-efficacy' on the deleterious effects of childhood maltreatment on trait anxiety in a genotype-dependent fashion. Specifically, the highest trait anxiety scores were observed in carriers of the less active NPSR1 rs324981 A allele with a history of childhood maltreatment and low general self-efficacy. However, when general self-efficacy was high, this pattern was reversed, with A allele carriers then showing the lowest trait anxiety scores despite the experience of childhood adversity. In individuals homozygous for the more active NPSR1 rs324981 T risk allele, increases in trait anxiety as a function of childhood adversity were observed to be largely unaffected by general self-efficacy. These findings suggest that in interaction with childhood adversity the TT risk genotype - irrespective of the influence of general self-efficacy increases the risk for anxiety, whereas the A allele does not, unless accompanied by low general self-efficacy, i.e. insufficient coping ability. Therefore, it can be proposed that high general self-efficacy is able to alleviate trait anxiety in A allele carriers despite a history of maltreatment, whereas low general self-efficacy can lead to increased anxiety scores in individuals with an otherwise equal genetic and environmental risk constellation.

\section{Contextual embedding}

The present results are in line with the existing $\mathrm{G} \times \mathrm{E}$ literature linking the NPSR1 rs324981 TT genotype to increased anxiety sensitivity $^{18}$ or heightened amygdala activation ${ }^{20}$ via environmental variation. Furthermore, they are in accordance with previous findings of the T allele mediating panic disorder as a nosological entity ${ }^{6}$ as well as intermediate phenotypes of panic disorder such as dimensional anxiety, psychophysiological responding, ${ }^{6,7}$ altered neural activation patterns ${ }^{6,9,10}$ or neuroendocrine stress responsiveness, ${ }^{8}$ whereas the A allele was generally not found to predispose to anxiety disorder risk per se or to influence anxiety traits on an intermediate level. The present $\mathrm{G} \times \mathrm{E} \times \mathrm{C}$ finding might furthermore 
(a)

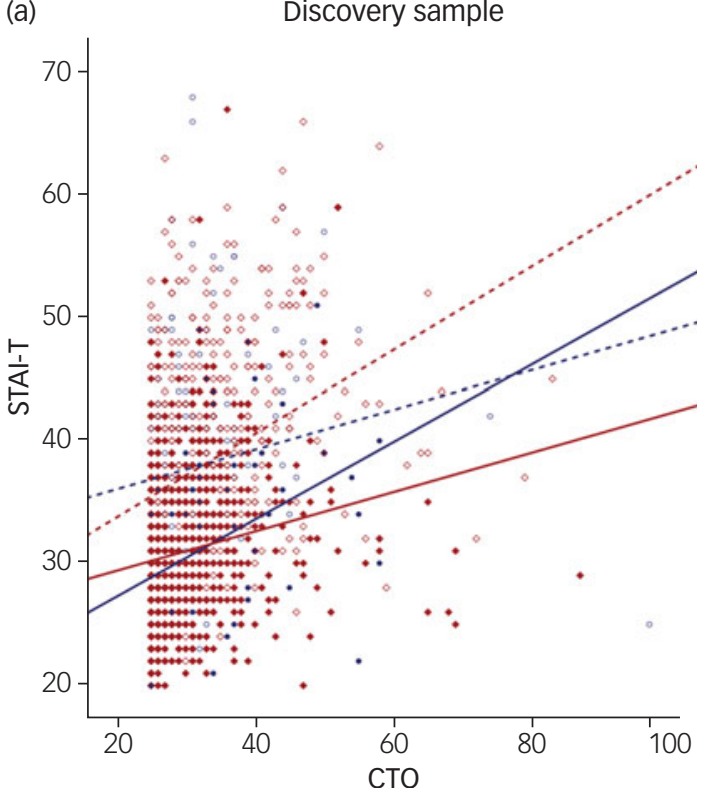

(b)

70

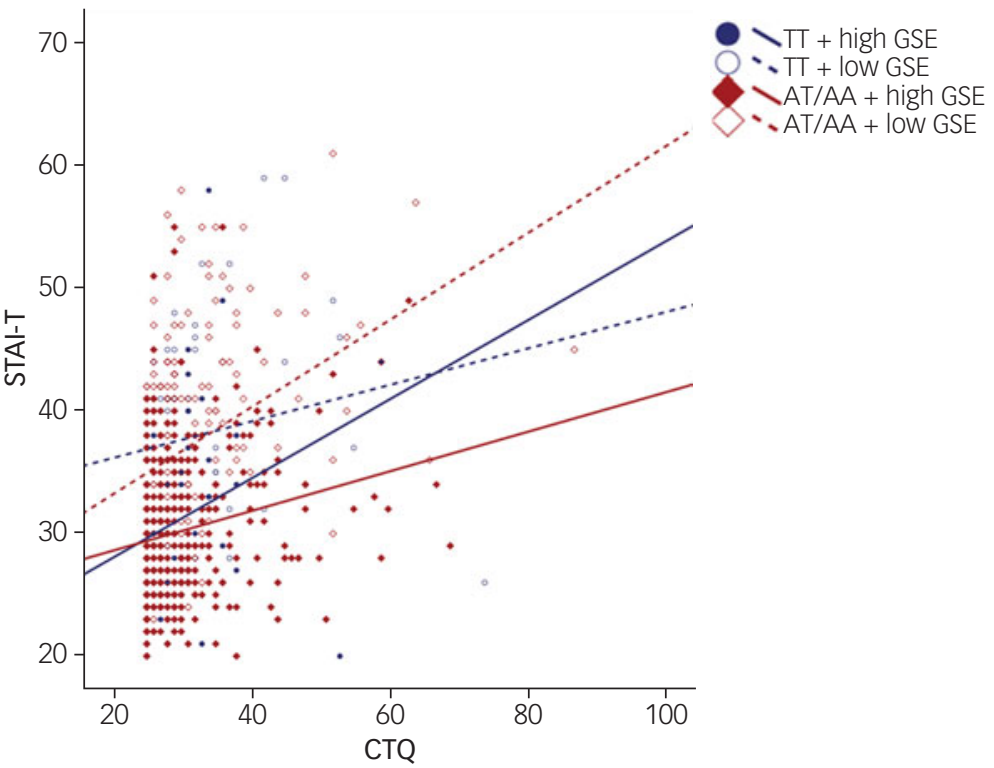

Fig. 1 Effect of childhood trauma on anxiety as a function of grouped NPSR1 rs324981 genotype and general self-efficacy.

(a) In the discovery sample of 1403 healthy probands, and (b) in the replication sample of 630 healthy probands (illustration of linear regression analyses).

CTQ, Childhood Trauma Questionnaire; GSE: General Self-Efficacy Scale; STAI-T, trait scale of the State-Trait Anxiety Inventory; neuropeptide S receptor (NPSR1) rs324981 genotype groups (TT genotype versus A allele carriers).

reconcile an apparently discrepant previous observation of the A allele being associated with a higher frequency of anxiety disorders in women reporting a negative family environment and with higher trait anxiety dependent upon the experience of past stressful life events: ${ }^{19}$ coping characteristics could function as an additional and previously unconsidered dimension able to buffer or, if maladaptive, further increase disease risk. Thus, in line with the 'differential susceptibility hypothesis, ${ }^{27}$ rather than representing a 'risk gene', NPSR1 appears to constitute a 'plasticity gene' insofar as its rs324981 A allele appears to drive increased susceptibility to environmental conditions, positive as well as negative, whereas the TT

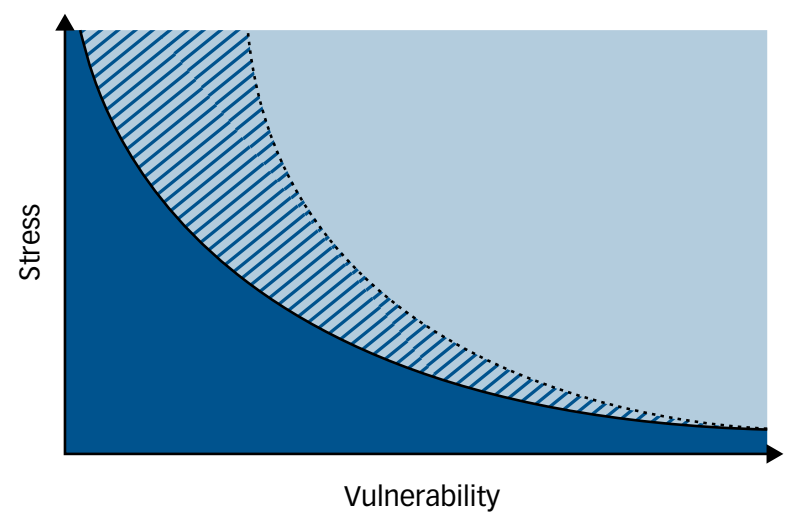

Fig. 2 An extended three-dimensional 'vulnerability-stresscoping model' of mental disorders based on the vulnerability-stress model by Zubin \& Spring.

The light blue area indicates illness, the dark blue area indicates wellness. Black lines indicate thresholds between illness and wellness. Coping ability can either decrease the threshold towards illness (solid black line) if low, or increase it (dotted black line) if high. The dark blue/light blue hatched areas indicate wellness or illness depending on an individual's constellation of vulnerability factors, stress experiences and coping ability. genotype may be less responsive to the resilience-increasing effects of adaptive coping capabilities.

\section{Implications}

Conceptually, the present study suggests revisiting the original concept proposed by Zubin \& Spring ${ }^{1}$ and to officially extend the general understanding of the 'vulnerability-stress model' in mental disorders by the dimension of coping towards a threedimensional 'vulnerability-stress-coping' or $\mathrm{G} \times \mathrm{E} \times \mathrm{C}$ model, where in the face of environmental adversity, impaired coping ability lowers the threshold towards illness despite a low genetic vulnerability, whereas high coping ability can buffer the impact of a high genetic and environmental factor constellation and thus increase the threshold towards illness (see Fig. 2).

From a clinical point of view, in contrast to an individual's genetic makeup and past adverse experiences, coping ability, i.e. general self-efficacy, is dynamic and malleable, and may therefore constitute a target for indicated preventive interventions in at-risk individuals increasing the threshold between wellness und illness. Indeed, general self-efficacy has been shown to increase following stress management training in university students ${ }^{28}$ and self-management interventions in severe mental disorders ${ }^{29}$ as well as over the course of cognitive-behavioural therapy. ${ }^{30}$ General self-efficacy was furthermore shown to confer clinical symptom improvement in panic disorder ${ }^{30}$ and social anxiety disorder. ${ }^{31,32}$

\section{Limitations and future directions}

The present study holds several strengths, such as investigating a functional genetic variant in large, sufficiently powered and independent samples of comparable demographic composition, under application of identical inclusion/exclusion criteria and using the same psychometric instruments and analysis methods under identical laboratory conditions, thus allowing for robust replication of the main finding. Nonetheless, some limitations should be taken into account while interpreting the present results and when 
designing future studies. The presently assessed samples comprised a relatively young, mostly student population of healthy volunteers with a mean age of 25.6 and 25.0 years, and may thus not be representative of the general population. Therefore, replication of the presently reported results is warranted interrogating more heterogeneous samples of healthy probands as well as extending the presently applied $\mathrm{G} \times \mathrm{E} \times \mathrm{C}$ approach to a clinical context by including high-risk individuals and patients with manifest anxiety disorders. In addition, in the present study childhood maltreatment was assessed retrospectively, which may be sensitive to recall bias.

The reported rates of experiences of childhood maltreatment were relatively low as indicated by low CTQ sum scores.

Extending the present cross-sectional design, longitudinal studies could provide promising insights into the developmental trajectories of anxiety from susceptibility to disorder manifestation. This approach could additionally contribute to the understanding of resilient functioning given evidence for the differential importance of environmental contributions during childhood, adolescence and adulthood, with the influence of environmental factors on the phenotypic stability of symptoms of anxiety and depression increasing with advancing age. ${ }^{33}$ Finally, on a genetic level, beyond the single candidate-gene approach future research may want to address the $\mathrm{G} \times \mathrm{E} \times \mathrm{C}$ model under consideration of haplotype or epistatic genetic effects as well as in the context of genome-wide association studies in sufficiently powered samples. This is because, in particular, recent genome-wide studies have reported several loci to significantly contribute to coping and resilience phenotypes. ${ }^{34,35}$ Along these lines, future studies will have to disentangle the individual and interactive genetic effects on risk and resilience, respectively, given that resilience towards stress-related mental disorders has been reported to show a heritability of up to $40 \%{ }^{36}$ and to display distinct biological underpinnings which do not simply constitute a reversal of mechanisms leading to disease risk. ${ }^{37,38}$ In addition, given increasing evidence for a role of temporally dynamic epigenetic processes in the modulation of gene function partly mediating adaptation to environmental influences in the context of anxiety disorders and anxiety-related phenotypes, ${ }^{39}$ future $\mathrm{G} \times \mathrm{E} \times \mathrm{C}$ models would benefit from the inclusion of epigenetic markers such as DNA methylation towards the formulation of a comprehensive multidimensional model of anxiety risk.

\section{Summary and conclusion}

In conclusion, the present $\mathrm{G} \times \mathrm{E} \times \mathrm{C}$ study exemplarily suggests that adaptive coping ability - i.e. high levels of general self-efficacy - may act as a broker between an otherwise detrimental gene-environment vulnerability profile and the resulting phenotype by exerting a protective effect in a resilience-enhancing way. In detail, a history of childhood maltreatment may result in increased trait anxiety and thus in an elevated risk for the development of anxiety disorders in NSPR1 A allele carriers, but only in the absence of the ability to successfully cope with adversity, whereas NSPR1 TT homozygotes carry an increased risk of anxiety traits as a function of maltreatment experiences irrespective of coping ability. The present results provide a framework for an extended 'vulnerability-stresscoping model' of anxiety and possibly mental disorders in general and - along the notion of a 'precision medicine' approach might allow for targeted preventive interventions for at-risk individuals carrying a $\mathrm{G} \times \mathrm{E}$ risk factor constellation by strengthening their adaptive coping strategies.

Miriam A. Schiele (D), PhD, Department of Psychiatry and Psychotherapy, Medical Center - University of Freiburg, Faculty of Medicine, University of Freiburg, Germany: Katharina Herzog. MSC, PhD, Department of Psychology (Biological Psychology, Clinical Psychology, and Psychotherapy), and Center of Mental Health, Julius-MaximiliansUniversität Würzburg, Germany; Leonie Kollert, MSc, PhD, Department of Psychiatry,
Psychosomatics and Psychotherapy, University of Würzburg, Germany;

Christoph Schartner (D, PhD, Department of Physiology University of California San Francisco, USA; and Department of Psychiatry, Psychosomatics and Psychotherapy, University of Würzburg, Germany; Elisabeth J. Leehr, PhD, Department of Psychiatry and Psychotherapy, University of Münster, Germany; Joscha Böhnlein, MSC, PhD, Department of Psychiatry and Psychotherapy, University of Münster, Germany; Jonathan Repple, MD, Department of Psychiatry and Psychotherapy, University of Jünster, Germany; Karoline Rosenkranz, MSc, Institute for Systems Neuroscience, University Medical Center Hamburg-Eppendorf, Germany; Tina B. Lonsdorf, PhD, Institute for Systems Neuroscience, University Medical Center Hamburg-Eppendorf, Germany; Udo Dannlowski, MD, PhD, Department of Psychiatry and Psychotherapy, University of Münster, Germany; Peter Zwanzger, MD, kbo-Inn-Salzach-Klinikum; and Department of Psychiatry and Psychotherapy, Ludwig-Maximilians-University of Munich, Germany; Andreas Reif, MD, Department of Psychiatry, Psychosomatics and Psychotherapy, University of Frankfurt, Germany; Paul Pauli, PhD, Department of Psychology (Biological Psychology, Clinical Psychology, and Psychotherapy) and Center of Mental Health, Julius-Maximilians-Universität Würzburg, Germany; Jürgen Deckert, MD, Department of Psychiatry, Psychosomatics and Psychotherapy, University of Würzburg, Germany; Katharina Domschke (D), MA, MD, PhD, Department of Psychiatry and Psychotherapy, Medical Center - University of Freiburg, and Center for Basics in Neuromodulation, Faculty of Medicine, University of Freiburg, Germany

Correspondence: Katharina Domschke. Email: katharina.domschke@uniklinik-freiburg.de First received 1 Nov 2019, final revision 21 Feb 2020, accepted 11 Mar 2020

\section{Data availability}

The data associated with the manuscript is not currently publicly available.

\section{Acknowledgements}

We gratefully acknowledge the skilful technical support by $\mathrm{C}$. Gagel as well as the support in proband recruitment (discovery sample) of D. Schümann, L. Kürbitz and K. Holitschke supervised by T.B.L. and P.Z. K.D., M.A.S. and P.Z. are members of the Anxiety Disorders Research Network (ADRN), European College of Neuropsychopharmacology (ECNP)

\section{Author contributions}

M.A.S. performed the statistical analyses and wrote the first version of the manuscript. K.D. designed the study and drafted the manuscript together with M.A.S. The discovery sample was coordinated and in part recruited by M.A.S. under the supervision of K.D., J.D., A.R. and P.P. The replication sample was recruited by K.H., E.J.L., J.R., J.B. and K.R. under the supervision of K.D., J.D., U.D., T.B.L., P.P. and M.A.S. Genotyping analyses were performed by C.S. (discovery sample) and L.K. (replication sample) supervised by K.D., J.D. and A.R. Part of this work was included in the doctoral thesis of M.A.S. Authors M.A.S. and K.D. have full and ongoing access to all the data in the study and take responsibility for the integrity of the data and the accuracy of the data analysis. All authors have read and approved the final article.

\section{Funding}

This work was funded by the German Research Foundation (DFG) - project number 44541416 TRR 58, subprojects Z02 (to K.D., A.R., P.P., J.D., T.B.L., U.D.) and C02 (to K.D. and J.D.) and CRC 1193, subproject Z03 (to A.R.).

\section{Declaration of interest}

K.D., A.R., P.P., J.D., T.B.L. and U.D. report funding by the German Research Foundation (DFG) in relation to this study. J.D. reports grants from the European Union and the Bavarian Secretary of Commerce outside the submitted work.

An ICMJE form is in the supplementary material, available online at https://doi.org/10.1192/ bjp.2020.73.

\section{References}

1 Zubin J, Spring B. Vulnerability: a new view of schizophrenia. J Abnorm Psychol 1977; 86: 103-26.

2 Musci RJ, Augustinavicius JL, Volk H. Gene-environment interactions in psychiatry: recent evidence and clinical implications. Curr Psychiatry Rep 2019; 21: 81.

3 Rutten BPF, Hammels C, Geschwind N, Menne-Lothmann C, Pishva E, Schruers $\mathrm{K}$, et al. Resilience in mental health: linking psychological and neurobiological perspectives. Acta Psychiatr Scand 2013; 128: 3-20.

4 Zubin J, Steinhauer SR, Condray R. Vulnerability to relapse in schizophrenia. Br J Psychiatry Suppl 1992; 161: 13-8.

5 Reinscheid RK, Xu YL, Okamura N, Zeng J, Chung S, Pai R, et al. Pharmacological characterization of human and murine neuropeptide s receptor variants. J Pharmacol Exp Ther 2005; 315: 1338-45. 
6 Domschke K, Reif A, Weber H, Richter J, Hohoff C, Ohrmann P, et al. Neuropeptide $S$ receptor gene - converging evidence for a role in panic disorder. Mol Psychiatry 2011; 16: 938-48.

7 Beste C, Konrad C, Uhlmann C, Arolt V, Zwanzger P, Domschke K. Neuropeptide $S$ receptor (NPSR1) gene variation modulates response inhibition and error monitoring. Neuroimage 2013; 71: 1-9.

8 Kumsta R, Chen FS, Pape HC, Heinrichs M. Neuropeptide S receptor gene is associated with cortisol responses to social stress in humans. Biol Psychol 2013; 93: 304-7.

9 Dannlowski U, Kugel H, Franke F, Stuhrmann A, Hohoff C, Zwanzger P, et al. Neuropeptide-S (NPS) receptor genotype modulates basolateral amygdala responsiveness to aversive stimuli. Neuropsychopharmacology 2011; 36 : 1879-85.

10 Domschke K, Akhrif A, Romanos M, Bajer C, Mainusch M, Winkelmann J, et al. Neuropeptide $S$ receptor gene variation differentially modulates fronto-limbic effective connectivity in childhood and adolescence. Cereb Cortex 2017: 27 554-66.

11 Scott KM, McLaughlin KA, Smith DA, Ellis PM. Childhood maltreatment and DSM-IV adult mental disorders: comparison of prospective and retrospective findings. Br J Psychiatry 2012; 200: 469-75.

12 Bandura A. Self-efficacy: toward a unifying theory of behavioral change. Psychol Rev 1977; 84: 191-215.

13 Endler NS, Speer RL, Johnson JM, Flett GL. General self-efficacy and control in relation to anxiety and cognitive performance. Curr Psychol 2001; 20: 36-52.

14 Muris P. Relationships between self-efficacy and symptoms of anxiety disorders and depression in a normal adolescent sample. Pers Individ Dif 2002; 32 337-48.

15 Maciejewski PK, Prigerson HG, Mazure CM. Self-efficacy as a mediato between stressful life events and depressive symptoms. Differences based on history of prior depression. Br J Psychiatry 2000; 176: 373-8.

16 Rudy BM, Davis TE, Matthews RA. The relationship among self-efficacy, negative self-referent cognitions, and social anxiety in children: a multiple mediator model. Behav Ther 2012; 43: 619-28.

17 Schönfeld P, Brailovskaia J, Bieda A, Zhang XC, Margraf J. The effects of daily stress on positive and negative mental health: mediation through self-efficacy. Int J Clin Health Psychol 2016; 16: 1-10.

18 Klauke B, Deckert J, Zwanzger P, Baumann C, Arolt V, Pauli P, et al. Neuropeptide $S$ receptor gene (NPSR) and life events: $G \times E$ effects on anxiety sensitivity and its subdimensions. World J Biol Psychiatry 2014; 15 $17-25$

19 Laas K, Reif A, Akkermann K, Kiive E, Domschke K, Lesch K-P, et al. Interaction of the neuropeptide $\mathrm{S}$ receptor gene $A \mathrm{As}^{107}{ }^{10}$ le variant and environment: contribution to affective and anxiety disorders, and suicidal behaviour. Int $J$ Neuropsychopharmacol 2014; 17: 541-52.

20 Streit F, Haddad L, Paul T, Frank J, Schafer A, Nikitopoulos J, et al. A functional variant in the neuropeptide $S$ receptor 1 gene moderates the influence of urban upbringing on stress processing in the amygdala. Stress 2014; 17: $352-61$.

21 Schiele MA, Ziegler C, Holitschke K, Schartner C, Schmidt B, Weber H, et al. Influence of 5-HTT variation, childhood trauma and self-efficacy on anxiety traits: a gene-environment-coping interaction study. J Neural Transm 2016; 123: 895-904.

22 Sheehan DV, Lecrubier $\mathrm{Y}$, Sheehan $\mathrm{KH}$, Amorim $\mathrm{P}$, Janavs J, Weiller $\mathrm{E}$, et al. The Mini-International Neuropsychiatric Interview (M.I.N.I.): the development and validation of a structured diagnostic psychiatric interview for DSM-IV and ICD10. J Clin Psychiatry 1998; 59(Suppl 20): 22-33.
23 Bernstein DP, Fink L. Childhood Trauma Questionnaire: A Retrospective SelfReport (CTQ). Manual. Harcourt Brace \& Co, 1998.

24 Schwarzer R, Jerusalem M. General Self-Efficacy Scale. In Measures in Health Psychology: A User's Portfolio. Causal and Control Beliefs (eds J Weinman, S Wright, M Johnston): 35-7. NFER-NELSON, 1995.

25 Spielberger CD, Gorsuch RL, Lushene RE. Manual for the State-Trait Anxiety Inventory. Consulting Psychologists Press, 1970

26 Kraemer $\mathrm{HC}$, Blasey $\mathrm{CM}$. Centring in regression analyses: a strategy to prevent errors in statistical inference. Int J Methods Psychiatr Res 2004; 13 $141-51$

27 Belsky J, Jonassaint C, Pluess M, Stanton M, Brummett B, Williams R. Vulnerability genes or plasticity genes? Mol Psychiatry 2009; 14: 746-54.

28 Molla Jafar H, Salabifard S, Mousavi SM, Sobhani Z. The effectiveness of group training of CBT-based stress management on anxiety, psychological hardiness and general self-efficacy among university students. Glob J Health Sci 2016; 8 : 47-54.

29 Lean M, Fornells-Ambrojo M, Milton A, Lloyd-Evans B, Harrison-Stewart B, Yesufu-Udechuku $A$, et al. Self-management interventions for people with severe mental illness: systematic review and meta-analysis. Br J Psychiatry 2019; 214: 260-8.

30 Gallagher MW, Payne LA, White KS, Shear KM, Woods SW, Gorman JM, et al. Mechanisms of change in cognitive behavioral therapy for panic disorder: the unique effects of self-efficacy and anxiety sensitivity. Behav Res Ther 2013; 51: 767-77.

31 Bouchard S, Gauthier J, Nouwen A, Ivers H, Vallieres A, Simard S, et al. Temporal relationship between dysfunctional beliefs, self-efficacy and panic apprehension in the treatment of panic disorder with agoraphobia. J Behav Ther Exp Psychiatry 2007; 38: 275-92.

32 Gaudiano BA, Herbert JD. Self-efficacy for social situations in adolescents with generalized social anxiety disorder. Behav Cogn Psychother 2007; 35 : 209-23.

33 Nivard MG, Dolan CV, Kendler KS, Kan KJ, Willemsen G, van Beijsterveldt CEM, et al. Stability in symptoms of anxiety and depression as a function of genotype and environment: a longitudinal twin study from ages 3 to 63 years. Psychol Med 2015; 45: 1039-49.

34 Maul S, Giegling I, Fabbri C, Corponi F, Serretti A, Rujescu D. Genetics of resilience: implications from genome-wide association studies and candidate genes of the stress response system in posttraumatic stress disorder and depression. Am J Med Genet B Neuropsychiatr Genet 2020; 183: 77-94.

35 Stein MB, Choi KW, Jain S, Campbell-Sills L, Chen CY, Gelernter J, et al. Genome-wide analyses of psychological resilience in U.S. Army soldiers. Am J Med Genet B Neuropsychiatr Genet 2019; 180: 310-9.

36 Waaktaar T, Torgersen S. Genetic and environmental causes of variation in trait resilience in young people. Behav Genet 2012; 42: 366-77.

37 Choi KW, Stein MB, Dunn EC, Koenen KC, Smoller JW. Genomics and psychological resilience: a research agenda. Mol Psychiatry 2019; 24: 1770-8.

38 Feder A, Fred-Torres S, Southwick SM, Charney DS. The biology of human resilience: opportunities for enhancing resilience across the life span. Biol Psychiatry 2019; 86: 443-53.

39 Schiele MA, Domschke K. Epigenetics at the crossroads between genes, environment and resilience in anxiety disorders. Genes Brain Behav 2018; 17: e12423. 